\title{
Construyendo un sentido en común dentro del área de gestión de recursos humanos de un hospital universitario en Bogotá
}

Building a common sense within the human resource management department of a university hospital in Bogota, Colombia

Recibido: diciembre 2 de 2010 | Revisado: febrero 13 de 2011 | Aceptado: febrero 28 de 2011

\author{
Hernán CAmilo Pulido-Martínez ${ }^{* * *}$ \\ LUZ MERY CARVAJAL-MARÍN \\ Pontificia Universidad Javeriana, Bogotá, Colombia
}

SICI: 1657-9267(201108) 10:2<595:SCAGRH>2.0.TX;2-9

Para citar este artículo. Pulido-Martínez, H. C. \& Carvajal-Marín, L. M. (2011). Construyendo un sentido en común dentro del área de gestión de recursos humanos de un hospital universitario en Bogotá. Universitas Psychologica, 10 (2), 595-607.

Este artículo es resultado del proyecto de investigación "El direccionamiento estratégico como marco para la producción de la salud, el trabajo y la subjetividad. El caso de las IPS en Bogotá" financiado por la Pontificia Universidad Javeriana. Este trabajo contó con la colaboración, como auxiliares de investigación, de las estudiantes: Rosy Janeth Hernández Orjuela, Jennifer Johana Espitia Vásquez, Luisa Fernanda Ángel Fortich, y Diana Lucía Jaimes Rivas.

** Departamento de Psicología, Cr 5 \# 39-00 piso 2, oficina 203.E-mails: cpulido@javeriana.edu.co; lmcarvaj@javeriana.edu.co

Research-ID: Carvajal-Marín, L. M., E-1383-2011.

\section{RES U MEN}

Alrededor del mundo, las organizaciones del sector salud se han tenido que adaptar a las prescripciones nacionales e internacionales que regulan tanto el trabajo como la salud. Este proceso ha sido acompañado de la introducción, en las áreas de recursos humanos, de conocimientos y prácticas guiadas por los principios provenientes de las Ciencias Sociales y Administrativas. En este encuentro entre las reformas liberales y las maneras de administrar la fuerza laboral, juegan un papel fundamental las significaciones que circulan en las organizaciones, puesto que de ellas depende, en gran medida, el éxito de agendas, programas e intervenciones dirigidas a la administración de los trabajadores. En este artículo, se presentan significaciones que están circulando acerca de trabajo, trabajador, organización, gestión humana y contratación, en un área de recursos humanos, compuesta por catorce trabajadores, de un hospital universitario en la ciudad de Bogotá, Colombia. El diseño cualitativo utilizado buscó recoger tanto las significaciones circulantes como su correlato emocional.

Palabras clave autores

Administración de recursos humanos en salud, significado del trabajo, Psicología del trabajo, investigación cualitativa en organizaciones.

Palabras clave descriptores

Psicología ocupacional, gestión del personal, contratación, relaciones laborales, actitud laboral, comportamiento de grupo.

\section{A B S T R A C T}

Around the world, healthcare organizations have had to accommodate to national and international regulations governing work and health. This process has been accompanied by the introduction of knowledge and practices coming from social and administrative sciences. In this encounter between the liberal reforms and the particular ways of managing the workforce, the circulating meanings play an important role because, to a large extent, the agendas, programs and interventions geared to towards workforce management depend on these circulating meanings to succeed. In this paper, meanings about work, worker, organization, human resources management and hiring that are currently circulating in a human resources department of a university hospital in Bogotá, Colombia, are presented. The qualitative design used in this paper aimed to collect the circulating meanings as well as their emotional correlative elements.

Key words authors

Human Resources Management, Meaning of Work, Work Psychology, Qualitative Research in Organizations.

Key words plus

Occupational Psychology, Personnel Management, Recruitment, Labour Relations, Work Attitudes, Group Behaviour. 


\section{El problema}

El sector de la salud en Colombia, al igual que en muchos otros países de la región, ha venido siendo objeto de radicales transformaciones durante las últimas décadas (Abrantes, Almeida, Rovere \& Salgado, 2010; Arroyo, 2002; De Roux, 2010). En el país, la puesta en marcha de la ley 100 de 1993 ha constituido un marco legal y práctico dentro del cual las organizaciones que ofrecen el servicio de salud han tenido que adaptarse a unas nuevas condiciones que, a diferentes niveles, afectan su funcionamiento. Esta ley, de acuerdo con VegaRomero (2008), ha favorecido la introducción, de manera masiva, de capital privado dentro del sistema, resultando en una situación caracterizada por lo que ahora puede ser llamado el negocio de la salud.

El manejo de la fuerza laboral, por supuesto, no ha sido una cuestión ajena a estos cambios, aún más, se puede decir que una cuestión central para el funcionamiento del sistema implica la transformación de la relación que se establece entre las organizaciones prestadoras del servicio de salud y sus trabajadores. En este panorama de cambio, y siguiendo el camino de otros sectores de la producción, en Colombia, han ido creciendo y cimentándose departamentos de recursos humanos en las organizaciones ahora conocidas como Instituciones Prestadoras del Servicio de Salud (IPS) (Calderón-Hernández, Naranjo-Valencia, Álvarez-Giraldo, 2006; García-Álvarez, 2006).

A la par de este incremento de la gestión de recursos humanos alrededor de departamentos especializados, en la literatura han empezado a aparecer señalamientos relacionados con problemas para el manejo de la fuerza laboral dentro del sistema de salud (Ruiz, Matallana, Amaya, Vásquez, Parada $\&$ Piña, 2008), los cuales tienen o tendrán que ser enfrentados, en el inmediato futuro, desde las áreas de gestión del personal (en algunos casos llamadas de talento humano). Por ejemplo, como señala Vega-Romero (2008), el "acto médico" ha cambiado, es decir, las relaciones del trabajo entre el personal asistencial y los pacientes son ahora distintas, en el sentido de que existe una nueva serie de restricciones que se imponen "por las organizaciones y los negocios que orientan la consulta" ( $\mathrm{p}$. 13). En esta misma línea, las organizaciones han tenido que lidiar, contener y, en algunos casos, fomentar los cambios que traen las nuevas formas de flexibilización laboral, tales como los contratos que se establecen entre las organizaciones que prestan el servicio de salud y las cooperativas de trabajo asociado, las cuales ofrecen prácticamente "manos y mentes" sin que medie un contrato permanente de trabajo.

Como en otros sectores que tienen una tradición de manejo de recursos humanos basados en las propuestas formuladas desde las ciencias administrativas y sociales, en el sector de la salud se ha formulado que su rol está, en gran parte, relacionado con los cambios organizacionales que conducen a la optimización de su funcionamiento. Este papel, por supuesto, no es nuevo. Desde la institucionalización de las disciplinas que lidian con el manejo de los seres humanos en el trabajo, y que se han hecho presentes como parte de los saberes que se aplican en intervenciones que se realizan para la gestión del personal, se ha propuesto que su rol está en, gran medida, relacionado con los cambios organizacionales, de los cuales se supone resultan exitosos o no, dependiendo de la anuencia que los miembros den a ciertos tipos de prescripciones para el actuar organizacional (Legge, 2005; Mayo, 1977; McWilliams, Flete \& Wright, 2001; PulidoMartínez, 2010a; Urdaneta-Ballen, 2006; Viteles, 1932). Sin embargo, de acuerdo con las características del sector de la salud, tal como señalan Ramanujam y Rosseau (2006, p. 811), las áreas de gestión humana han tenido que poner de presente, para establecer un lugar dentro de los sistemas de salud, que el éxito de las organizaciones de salud no depende exclusivamente de la cuestión clínica sino que también "los retos que se enfrentan son organizacionales".

Que los retos que se enfrentan en las organizaciones que ofrecen el servicio de salud también sean organizacionales, de por sí, no significa que las actividades que se realizan en otros sectores de la producción, se puedan extender mecánicamente al sistema de salud. Están bien documentadas las 
particularidades que se deben considerar en términos de políticas y prácticas de personal realmente pertinentes para la gestión de los recursos humanos en el sector de la salud (Matallana, Amaya \& Vásquez, 2008; Miller-Franco, Bennett \& Kanfer, 2002). En este sentido, en el país, ya ha sido señalado, no solamente para el sector de la salud, que la investigación debe continuar pues poco se ha indagado acerca de los discursos y prácticas, de las adaptaciones e hibridaciones del conocimiento relacionado con la gestión del personal que se está utilizando, así como poco se han considerado las implicaciones que han traído las intervenciones y transformaciones relacionadas con las prácticas cotidianas organizacionales, para el manejo y administración de la fuerza laboral (Dávila, 1985; Pulido-Martínez, 2009).

Si bien no se pueden hacer extensiones inmediatas al sector de la salud, tampoco se puede evitar estar vigilantes sobre las problemáticas que han sido señaladas por diferentes investigaciones, acerca de los departamentos en donde se adelanta la gestión de los recursos humanos. La literatura crítica al respecto ha llamado la atención sobre la carencia de un marco conceptual complejo que dé coherencia a las distintas tareas que se llevan a cabo como parte de la gestión del recurso humano. Cuestión que ha dado como resultado que en las áreas de gestión de personal se asuma una posición reactiva más que propositiva ante los problemas administrativos, pues ante la falta de unas premisas conceptuales se "reacciona" con soluciones a los problemas del acontecer organizacional, generando un lugar que ha sido calificado de "ambiguo" entre la gerencia y los trabajadores (Garavan, O’Donnell, McGuire \& Watson, 2007; Holton, 2003).

Se ha reconocido cómo las significaciones relacionadas con el trabajo están implicadas en cada aspecto de la práctica del desarrollo de los recursos humanos (Flesher, 2009; Pulido-Martínez, 2010b). A tal punto que se puede afirmar que en muchas situaciones de la cotidianidad organizacional las significaciones proporcionan sentidos a las prácticas (Schvarstein, 2003). Las significaciones con respecto al trabajo cu- bren una gama amplia de aspectos que comprende, de acuerdo con Flesher (2009), desde la relevancia que se le atribuye a ciertas formas y áreas de capacitación hasta las atribuciones y consecuentes planes de acción, basados en la retroalimentación que se obtiene de las encuestas con los empleados.

Sin embargo, se presenta, en el país, no solamente dentro del sector de la salud, un desconocimiento generalizado de los significados que circulan en las áreas de gestión de humana. Este desconocimiento generalizado apunta hacia una carencia central, que se infiere de los reportes de la literatura (Garavan et al., 2007), que se desconoce en gran medida cómo se están constituyendo los regímenes administrativos en las organizaciones de salud, en las condiciones que están afectando el sector.

Así, dentro de este panorama, este estudio se preguntó por las significaciones que circulan acerca del trabajo, del trabajador, de la organización, del área de gestión humana y de la organización en el escenario de un área de recursos humanos de una institución hospitalaria universitaria, de cuarto nivel, de carácter privado, en la ciudad de Bogotá.

\section{Significados}

Contrariamente a las visiones de corte positivista que consideran que las organizaciones se comportan como "máquinas racionales", donde el lenguaje permite hacer descripciones neutrales de las situaciones que se viven cotidianamente, las perspectivas constructivistas hacen énfasis en que las organizaciones son campos en donde se llevan a cabo interacciones en las cuales básicamente se negocia la cotidianidad organizacional, a través del lenguaje (Gergen \& Thatchery, 1996; Morgan, 1990). La negociación de las significaciones, y por tanto, la prevalecía y permanencia de unas sobre otras, corresponde directamente a las relaciones de poder que se hacen patentes en el mundo del trabajo (Gillespie, 1993; Schvarstein, 2003; Stecher, 2010).

En referencia al sector de las salud, como en los demás sectores que entran contemporáneamente 
en el circulo de la globalización, las significaciones que circulan son el resultado de las relaciones de poder que resultan en la regulación internacional del trabajo y de la salud, en las particulares apropiaciones que de estas regulaciones se hace en cada una de las organizaciones y en las interacciones que ocurren en el día en y entre los grupos particulares, que componen la estructura organizacional (Pulido-Martinez, 2008; Schvarstein, 2003). Por tanto, como sugiere García-Álvarez (2008), las significaciones o apropiaciones particulares que realizan los miembros de las organizaciones del sector de la salud permiten entender la "lógica" de las instituciones que ofrecen el servicio de salud, en tanto que esta "apropiación" que ocurre en el nivel interpersonal, no es independiente de las prescripciones formuladas por las orientaciones neoliberales, que determinan la estructura y funcionamiento del sistema (p. 29).

En la determinación de las significaciones que circulan en las organizaciones interactúan las instituciones, las relaciones con otras organizaciones, las particularidades organizacionales, las relaciones grupales y las interacciones cara a cara. En otras palabras, en las condiciones actuales por las que atraviesa el sector de la salud, en las significaciones que circulan en las organizaciones se reflejan las influencias globales y locales que moldean las formas en las cuales se trabaja.

Por otra parte, se supone que, si bien las concepciones acerca del acontecer organizacional que circulan entre los miembros de un grupo organizacional no garantizan directamente que las acciones se lleven a cabo en un sentido predeterminado por dichas concepciones, sí, al menos, se constituye un marco para el actual cotidiano y para que intervenciones organizacionales se puedan adelantar, en la medida en que es posible entrar en "sintonía" con significaciones circulantes (Cunliffe, 2003; Restrepo-Jiménez, 1998). Este, por llamarlo de alguna manera, "mínimo" compartido de significación, resulta fundamental, tanto a la hora de entender las maneras en las cuales los ámbitos de trabajo fluyen o por el contrario se estancan y, por tanto, es muy útil al momento en que se hace necesario proponer los caminos siguientes dentro de las organizaciones (Pulido-Martínez \& CarvajalMarín, 2005; Schvarstein, 2003).

En este estudio, por supuesto, no se buscaba hacer generalizaciones o recoger de manera exhaustiva las versiones que circulan en el sector. Por el contrario, en la medida en que los departamentos de recursos humanos están constituidos por una diversidad amplia de profesiones y oficios, se buscaba, en el caso específico de una IPS, hospital universitario de cuarto nivel, describir las versiones que circulan entre sus miembros para contribuir a la comprensión de las maneras en las cuales las significaciones que circulan hacen una contribución central a las formas de construir los regímenes administrativos, que se requieren acordes con las condiciones del sector.

Entonces, dentro del contexto los cambios que ocurren en el sector de la salud, el estudio estuvo dirigido a explorar las significaciones de trabajo, trabajador y organización que circulan en un área de recursos humanos de uno de los principales hospitales universitarios del país. Con el supuesto de que las significaciones escogidas son potencialmente centrales para guiar el actuar de los miembros de las áreas de recursos humanos, la pregunta se formuló así: ¿Qué significados circulan acerca del trabajo, del trabajador, de la organización, del área misma de recursos humanos y de la cuestión salarial en las condiciones laborales contemporáneas que se imponen para el sector salud dentro de un hospital?

Para dar respuesta a esta pregunta, se entrevistaron a profundidad los catorce miembros del área de gestión humana del hospital universitario. La entrevista no estructurada contó con un guión que hacía referencia a las categorías que se querían indagar. Una vez recogida la información, se llevaron a cabo dos fases. En la primera, los investigadores clasificaron las diferentes versiones que emergieron, a través de matrices de sentido, allí se ubicaron y analizaron las diversas versiones que surgieron acerca del trabajo, del trabajador, de la organización, del área de recursos humanos y de los salarios. Enseguida, se seleccionaron fragmentos significativos que daban cuenta de la amplia variedad de versiones que aparecieron. En 
la segunda fase, a través de un taller especialmente diseñado, se trabajó con los participantes en la interpretación de los datos. Para esto, se proporcionaron a los miembros del área los fragmentos escogidos, los cuales fueron acomodados en diversos paquetes de acuerdo con cada una de las categorías previamente establecidas. Se les pidió entonces a los participantes que construyeran un texto de cualquier género literario que reflejara, de acuerdo con los fragmentos y con la experiencia propia los conceptos de trabajo, trabajador, organización, área de recursos humanos y salarios, para presentarlo al grupo en conjunto. Se hizo énfasis en que se podían tomar palabras, frases o fragmentos completos, haciendo los cambios y selecciones que consideraran pertinentes, para incrementar el significado de lo que querían expresar.

Hay que aclarar de antemano que aquí se presentan solamente las construcciones que realizaron los miembros del área de recursos humanos a partir de la información recolectada. Se tomó esta decisión pues, como afirma Reilly (2010) en diversos estudios cualitativos, los resultados de los datos analizados desde la perspectiva de los investigadores y de los investigados, son bastante similares. No obstante, de acuerdo con lo reportado en la literatura en la interpretación llevada a cabo por los participantes, una dimensión profundamente emocional emergió, cuando se les pidió que construyeran un nuevo texto.

En esta manera de proceder para interpretar los datos, es pionero el trabajo de construcción, dentro del género literario de la poesía, con fragmentos del acontecer organizacional adelantado por Rosemary Reilly (2010). Se busca conducir la investigación, aumentar los criterios que hacen confiable la investigación cualitativa y que se diferencian de los tradicionales criterios de validez y objetividad de la investigación cuantitativa (Cho \& Trent, 2006). Por ejemplo, se aumenta la credibilidad, pues las construcciones que representan la realidad provienen directamente de los investigados; se incrementa la coherencia, en la medida en que encuentran focos que articulan la significación; se aumenta el consenso, a través de la revisión de los datos, las experiencias y los sentimientos de los participantes y, además, se abre un espacio para que los miembros de la organización propongan una imagen que es socialmente aceptable para ser publicada en los medio especializados (Cho \& Trent, 2006).

En este estudio, no se pidió la construcción de un texto dentro de un género específico. Esta decisión se tomó esperando que la elección misma del formato del texto formara parte del análisis y agregara valor a la dimensión emocional que pudiera aparecer. Adicionalmente, en el pedido impreciso del género del texto, se buscó que se consolidaran criterios de confiabilidad que se han propuesto para la investigación cualitativa, tales como, mayor empatía a través de la reflexión conjunta que entre sí adelanten los miembros de la organización, de modo tal que se contribuye a la construcción de grupos, y a una conexión más cercana entre los investigados y los miembros del equipo de investigación.

A continuación, se presentan los textos construidos por los miembros del área de gestión humana, acompañados de notas que hacen referencia a análisis denotativos y connotativos de los mismos.

\section{Resultados}

\section{El trabajador}

La primera elaboración que se presenta refiere las versiones circulantes acerca del trabajador. Se quiere llamar la atención sobre el hecho de que el, si se puede llamar en este caso, género literario escogido para ilustrar lo que significa ser trabajador fue un aviso clasificado dividido en dos partes, o mejor dos avisos clasificados que se vuelven interdependientes y que señalan lo que la empresa busca y lo que el trabajador busca. Los dos avisos dejan entrever la dialéctica que se crea entre estas dos maneras de requerir diferentes aspectos de la vida organizacional, lo cual da como resultado una idea de trabajador que, como tal, no aparece en los datos tal como fueron codificados, pues esos se organizaron bajo el supuesto de que podían existir diferentes versiones del ser trabajador; sin embargo, en la codificación las versiones no necesariamente 
interactuaban, así como lo propone la interpretación que realizaron los miembros del área.

En primer lugar, el aviso clasificado hace referencia a las características generales del posible trabajador que la organización esta buscando para el área de recursos humanos y que, según dejaron entrever en el taller los participantes, es una imagen ideal no solamente para la gestión del área, sino para el hospital en su totalidad. La segunda parte señala aspectos centrales que requiere el trabajador, básicamente para estar bien, partiendo de lo que el trabajo en forma de empleo puede ofrecer.

Aviso clasificado No 1

\section{SE BUSCA:}

Una persona recursiva, proactiva, que cumpla con sus responsabilidades y con la institución, que dé soluciones, llena de valores tales como excelencia, proactividad, dinamismo y liderazgo.

\section{Aviso clasificado No 2}

\section{TRABAJADOR BUSCA:}

Un equipo de trabajo con el cual cumplir sus labores, donde se capacite y se satisfaga su deseo de aprender. Un lugar en donde pueda alcanzar una estabilidad, en el cual encuentre relaciones cordiales, donde se tengan en cuenta sus opiniones y las de los otros. Un lugar en donde pueda dar lo mejor para el servicio de los demás.

La descripción de las características del trabajador muestra al empleado contemporáneo y hace referencia a las condiciones laborales actuales sin las cuales no se puede proponer dicha construcción. En términos del mercado de trabajo, las formas de trabajar se caracterizan por la incertidumbre acrecentada, mayores índices de desempleo, "terciarización" de contratos y disminución de la responsabilidad que las organizaciones tienen con respecto a los trabajadores (Beck, 2000; Castel, 1997, 2004; Naredo, 2001). Si bien estas no son las condiciones que imperan para el área de gestión de recursos humanos, sí muestran su capacidad de generar significaciones que atraviesan los distintos ámbitos organizacionales y señalan la disposición que de antemano existan, para que se puedan colonizar condiciones de trabajo, que no directamente corresponden a esa flexibilización que ocurre.

En las condiciones de flexibilización se espera que el trabajador, hoy en día, no solamente cumpla con una serie de tareas circunscritas al manual de funciones. En la medida en que el trabajo se flexibiliza y que la manera de administrar se aleja del control directo y se aproxima al control de la subjetividad, este trabajador tiene que actuar por su cuenta sin que medie un ente o jefe que dé cuenta de su propio actuar (Alvesson \& Willmott, 2002; Walkerdine, 2005). Como el primer aviso clasificado reza, el trabajador tiene que dar cuenta de lo que hace y esto está vinculado con ese "excedente" que debe estar en capacidad de aportar a la organización más allá de lo que se pide. Este es un trabajador que se conduce a través de la administración de sus fuerzas internas, para obtener una "movilización" constante que se traduce como autonomía (Rose, 1992, 1999). Autonomía que es considerada en términos tales como recursividad, proactividad, dinamismo y liderazgo. Como se ha reportado en la literatura, el trabajador "quieto" implica que no está acorde con los tiempos que corren, en donde se debe abandonar las "zonas de comodidad", que implican no hacer esfuerzos adicionales; estar estático significa estar sin goce y por tanto implica no alcanzar la satisfacción que debe provenir de la colaboración con la organización (Pulido-Martínez, 2010a).

Contrasta la segunda parte del aviso clasificado con lo que el trabajador busca. Aquí la cuestión de las condiciones laborales emerge como punto central. Estabilidad, capacitación, relaciones cordiales, reconocimiento y posibilidad de hacer aportes en términos de servicio a los demás. En otras palabras, se constituyen dos aspectos centrales de lo que significa ser trabajador, autonomía y movilización por parte de la empresa, frente a bienestar en diversas dimensiones por parte del empleado. Incertidumbre vs. certidumbre muestran los polos de significación, dentro de los cuales se está construyendo el trabajador del área de gestión humana. 


\section{El trabajo}

La composición en el género canción fue escogida para reflejar las versiones circulantes del trabajo. Específicamente, los participantes hicieron una parodia de una de las canciones más conocidas de la música bailable.

La experiencia propia de trabajar se refleja en la canción, constituyendo el centro de la significación. Se refleja una ética, o mejor una visión o valoración particular, de la satisfacción que se obtiene del trabajo en un sitio particular. En el taller, cuando se presentó el texto, los participantes cantaron las estrofas escritas, añadiendo dimensiones emocionales vinculadas con la alegría de trabajar en el hospital.

¡Ay! Que agradable es trabajar

¡Ay! Cuando llegamos al Hospital

Me gusta levantarme en la mañana

Tomar el baño y salir a trabajar

Pensar en las cosas buenas que allí pasan

Y en todas las demás que hay por realizar

¡Ay! Que bonito es el Hospital

Sus personas y demás

Todos llegando saludando y

De inmediato a trabajar

Aprender, ser responsables

Ser personas de triunfar

iAy! Entre amigos y familia

En el Hospital crecerás

iAy! iAyayay!

Qué bonito que en el Hospital

Sus equipos de gestión

Calidad y compromiso

Son su gran realización

¡Ay! iAyayay!...

Por supuesto, no se pueden desprender las versiones circulantes del trabajo de las del trabajador, pues constituyen dos ramas de lo que significa trabajar. Aquí en la canción se encuentran entrecru- zadas. Sin embargo, surgen ciertas particularidades. Es llamativo que el trabajo no se asocie a otra cuestión diferente del goce. Existen en el mundo del trabajo multitud de posibilidades de significaciones circulantes relacionadas con el empleo, tales como, fuente del sustento, generador de cierta identidad profesional y portador de virtudes, las cuales resultaban importantes hasta hace relativamente poco tiempo. Sin embargo, como bien aparece aquí, el placer al trabajar se ha tornado en la cuestión central. Este placer se asocia a aspectos psicológicos relacionados con las relaciones, la realización personal, la responsabilidad de tal manera que se vaya hacia "ser personas de triunfar".

Se podría entonces decir que el bienestar psicológico caracteriza el trabajo en el área de gestión. Las condiciones materiales han pasado así a no tener un lugar. Quizá este se relaciona con la deseabilidad social que, en el medio colombiano, poco admite que se hable en espacios colectivos, por ejemplo de la remuneración como parte del significado de trabajar.

\section{El área de gestión humana}

El cuento fue el género escogido para organizar las significaciones con respecto al área de gestión humana. Acorde con esta forma, presenta una introducción, un nudo y un final inconcluso.

Un pasado.... un presente y un fulano

Había una vez un departamento de gestión humana en el que se perdían los documentos, en el que no se solucionaban los problemas del personal del hospital, en el que las puertas estaban cerradas y al que la gente temía asistir.

Poco a poco este departamento ha ido cambiando, se han organizado los procesos, los miembros del equipo se involucran con la gente del hospital y con sus familias, brindando solución a sus problemas, hablando siempre una voz de aliento, sintiendo y haciendo sentir a los demás que el hospital es su segundo hogar. 
Ahora gestión humana ha asumido un nuevo rol, buscando el bienestar de todas las personas, en un ambiente laboral agradable y velando por el logro de los objetivos organizacionales.

A pesar de que por dar demasiado de nosotros a los demás hemos ido descuidando nuestro equipo estamos en la mira de todo el hospital, somos una red que lucha por borrar la imagen negativa que se construyó durante muchos años.

Y colorín colorado este cuento no ha terminado...

La forma escogida señala la idea de proceso en el cual es posible mirar el pasado, cavilar sobre el presente y dejar preguntas para el futuro. El cuento, por sus características, facilita que las significaciones se organicen alrededor del cambio.

Vale la pena resaltar que, si bien en relación con el trabajador la idea de movilidad hacia actividades es central, aquí el desplazamiento o la movilidad tienen otro sentido. El cambio trata de localizar la función de la gestión de los recursos humanos en la intersección entre la organización y los trabajadores. Como se había mencionado con anterioridad, las áreas de gestión de los recursos humanos están alcanzando este lugar dentro del sector de la salud, pues anteriormente se habían visto limitadas, en muchos casos, a adelantar procesos de gestión que estaban en directa relación con la nómina, la contratación y el despido. En la medida en que el régimen administrativo, en su evolución, da cabida a las prácticas y discursos, por ejemplo, de corte psicológico, el problema del cambio y el lugar del departamento de gestión humana en relación con el cambio, ocupan un papel central.

En términos generales Un pasado, un presente... y un fulano refleja la instauración de una nueva dirección administrativa para el personal, que va de la mano con las significaciones mayores circulantes plasmadas en los avisos clasificados y en la letra de la canción, considerados antes. Este régimen administrativo se desplaza del antiguo que se le caracteriza por su desorganización, falta de consideración por los otros y por el miedo que infundía.
Vale la pena resaltar también las implicaciones que vienen de la mano con la instauración de otras maneras de hacer la gestión. Los objetivos organizacionales y un ambiente de trabajo agradable traducen la relación entre la organización y el trabajador, respectivamente. Planteada así, esta relación corresponde a la visión que han propuesto para el trabajo la perspectiva humanista dominante en las Ciencias Administrativas y Sociales.

Sin embargo, la instauración de otras maneras de hacer la gestión y de lograr un puesto para el área que medie entre los trabajadores y la organización, se encuentra con el lugar "ambiguo", que se reporta en la literatura, que entre otras cosas dé cabida al escrutinio y critica, desde los diferentes grupos que componen la organización.

\section{El hospital}

El grupo que se encargó de esta categoría, para organizar sus fragmentos y agregando su experiencia particular, construyó un texto escrito en el género prosa:

Quince años atrás el hospital era un lugar donde los directivos y algunos personajes asistenciales no caminaban, solamente volaban como en un cuento de hadas.

Si se tenía alguna duda o se necesita ayuda se debía seguir el protocolo, como en el palacio de justicia.

Después todo fue cambiando paulatinamente.

El león una vez encerrado en la jaula se fue volviendo dócil y manso, la soberbia fue descendiendo y llegó el momento en donde el pastor se reúne con sus ovejas.

Y así se fueron concientizando que estaban compartiendo en un hospital, donde había enfermedad y dolor y donde era necesaria más humanidad.

Anteriormente, algunos indiferentes decían lo siguiente: yo no me estoy dando cuenta si en el hos- 
pital tienen mil enfermos, tampoco si no tienen en urgencias donde acomodar la gente.

A mí todo eso me resbala.

Otros que protestaban en cambio decían, a mí me ha gustado poder ayudar pues este es un hospital que se caracteriza por ser muy humano y me siento parte de él.

Algunos en la mitad y también aguafiestas opinaban, igual aquí yo trabajo como pueda, con las uñas, tampoco está todo perfecto ni tan mal, este no es el mejor hospital.

Hoy en día en el bosque se han roto las ataduras, los valores y la calidad humana se ven reflejados en la calidad profesional.

Sin embargo, siempre es bueno fortalecer algunos estándares en nuestro grupo de gestión humana para ser un verdadero equipo donde reine la sinceridad y la solidaridad.

Los principios humanistas son el más notorio articulador de las significaciones al respecto del hospital. Sobre estos principios se debe estar vigilante, para alcanzarlos. No hay que hacer mayores análisis de este texto pues parece hablar por sí mismo. Baste decir, como afirma Reilly (2010), que cuando los textos tienen algunas características poéticas y metafóricas, la fuerza que estos transmiten conecta directamente al lector con los investigados más que cualquier análisis que pueda ser hecho por los investigadores.

\section{La contratación}

Una noticia publicada en un diario dio lugar a la interpretación de las condiciones laborales:

Difícil tarea

Hoy en día es muy común la contratación del personal a través de las empresas de servicio temporal, las cuales han surgido como una solución para las empresas que requieren vincular a una persona para realizar una labor determinada y por un tiempo específico.

Sin embargo este tipo de contratación causa una notable insatisfacción a la persona vinculada por este medio debido a la continua comparación con sus compañeros que se encuentran vinculados directamente con la empresa, también se sienten en desventaja ya que no se sienten respaldados en el momento de requerir créditos bancarios, siempre tienen temor de enfrentarse a una terminación del contrato repentina, ya que con la naturaleza de esta contratación no reciben ningún tipo de indemnización.

Por otra parte se puede decir que el personal vinculado directamente por la empresa se siente completamente satisfecho, ya que al compararse con sus pares encuentran notorias diferencias saláriales en cargos y funciones similares.

De igual manera es común encontrar personas insatisfechas con su asignación salarial. Pero también para algunos no solo la remuneración económica es importante, sino que hay otros factores que intervienen en su motivación y satisfacción.

Entonces... iqué tienen que hacer los empleadores colombianos para mantener satisfechos a sus colaboradores?

El género noticia de por sí indica la novedad del asunto. La novedad se refiere básicamente a las nuevas condiciones laborales que atraviesa no solamente el sector de la salud, sino el mundo del trabajo en general. Algunas de las contradicciones que se tienen que enfrentar desde las áreas de gestión del recurso humano con la llegada, especialmente, de la precarización salarial, están puestas de presente.

La contratación se incluyó como uno de los aspectos por indagar de manera especifica, en razón de que se asumió la dificultad que se presenta en los participantes para expresarse sobre estos asuntos relacionados con el factor dinero de las 
condiciones laborales. A este respecto, es bueno anotar que tanto en los textos anteriores, como en los fragmentos significativos, la contratación y su derivado, las formas de remuneración, no fueron considerados.

La noticia describe con claridad diáfana qué es la precarización laboral con la incertidumbre que la acompaña. Allí, desde la perspectiva del trabajador, se presentan las dimensiones principales de estos procesos que han desmejorado la "condición salarial” y que amenazan con la desaparición de la forma empleo.

Pero no se detiene solamente en esto la noticia, la novedad abarca también las nuevas relaciones que se generan entre los trabajadores "terciarizados" y aquellos que están vinculados directamente por la organización. La división que se suscita, producto de la comparación entre "permanentes y temporales" o entre unas condiciones precarias y las ventajas que todavía ofrece el empleo permanente, hace valorar aún más las condiciones de contrato fijo y produce un tipo de satisfacción que, seguramente, está relacionada con la disminución de la incertidumbre. Esta disminución de la incertidumbre señala, de cierta forma, un es posible estar en peores condiciones haciendo lo mismo, asî como promueve conformidad con las condiciones salariales, pues es común encontrar personas insatisfechas con su asignación salarial.

De soslayo, se comenta que en la condiciones de empleo permanente existen otras motivaciones en relación con el trabajo, más allá de la remuneración. Esta referencia, dentro del contexto de la noticia, señala un aspecto fundamental para la gestión de los recursos humanos. Bien se conoce que los trabajadores precarizados que trabajan para empresas temporales, están motivados y son eficientes, la expectativa del empleo permanente en conjunto con la incertidumbre de la renovación de su contrato temporal, los mueve ha desempeñarse con eficiencia en la tarea (García-Álvarez \& Carvajal-Marín, 2007). En estas condiciones laborales, las propuestas hechas por las Ciencias Sociales para el manejo de los trabajadores, parecen naufragar. El ámbito del empleo permanente, amenazado constantemente por la precarización, representa el espacio cada vez más reducido, para la acción de las áreas de gestión humana, al menos en lo relacionado con la pertinencia de las herramientas convencionales para dar respuesta al manejo de los trabajadores. La pregunta con que se cierra la noticia representa de por sí un reto para las áreas de gestión del personal, ante las nuevas condiciones laborales que generan precarización y constante comparación entre los trabajadores iqué tendrán que hacer para mantener satisfechos a sus trabajadores?

\section{Conclusiones}

Las significaciones que circulan en el área de gestión humana del hospital que participó en este estudio, dan muestra del cambio que se está experimentando en el sector. A su vez, por tanto, son ejemplo de cómo las perspectivas que se proponen desde las Ciencias Administrativas y Sociales, están acompañando la transformación de las organizaciones que ahora tienen que adaptarse a diferentes tipos de prescripciones provenientes de la regulación internacional y nacional del mundo del trabajo y de la salud, a la cual se le llama contemporáneamente geocultura liberal (Papadopoulos, 2003).

Estos "mínimos de significación" de los que hablan los textos apuntan a las agendas, programas e intervenciones que les son y serán armónicos. Sin embargo, llama la atención la neutralidad de las significaciones en relación con la organización como prestadora de un servicio de salud. Pueden las significaciones que circulan en las áreas de gestión humana desligarse del hecho de que se está en medio de una organización que ofrece el servicio de salud, esta parece ser una pregunta pertinente, ante las significaciones encontradas.

Se está, entonces, invitando a reconsiderar el supuesto que atraviesa las perspectivas propuestas por las Ciencias Sociales y Administrativas con respecto a su conocimiento, del cual se supone es útil, y se comporta de manera análoga, aun en contextos bien disímiles. Qué de las particularidades de las maneras de conceptuar e intervenir la salud debe ser parte de las significaciones que atraviesan 
la gestión de recursos humanos y cómo deben estas incorporarse, son preguntas que deberán responderse en términos particulares.

Quizás, hasta hace poco, como señala Holton (2003), no existía un grupo de investigadores que se encargara de pensar en su conjunto los problemas relacionados con la gestión y sí, "históricamente", muchas apropiaciones y formulaciones derivadas de la práctica, y por supuesto llevadas a cabo por los practicantes profesionales que se desempeñan en las organizaciones. Es, entonces, tiempo de un trabajo conjunto que supere la tradicional división entre investigadores y profesionales, que parece más adecuada para la situación de los países noratlánticos que para las características de nuestro medio.

Por último, alguna consideración sobre la metodología escogida, la cual permitió establecer, no solamente significaciones, sino cómo estas se articulan a través de la interpretación conjunta que realizó el Grupo. La interpretación conjunta a partir de fragmentos de entrevistas para producir un nuevo texto, implica una jerarquización de las significaciones y una escogencia del género del texto que se utiliza para comunicarlas con mayor potencia emocional, las cuales tendrán que ser exploradas en mayor medida en otros estudios. Hay que anotar que la construcción conjunta, como era de esperarse, contribuyó a hacer menos asimétricas las relaciones de poder que surgen de los procesos investigativos, aun de aquellos que asumen una perspectiva cualitativa. Habrá, a este respecto, que explorar maneras de escritura conjunta que hagan más efectiva la horizontalización, por así decir, de las relaciones entre participantes e investigadores.

\section{Referencias}

Abrantes, R., Almeida, C., Rovere, M. \& Salgado, O. (2010, noviembre). Experiencia América Latina. México, Brasil, Argentina, Chile. Panel Latinoamérica. Primer Congreso Internacional de Sistemas de Salud iHacia un Nuevo Sistema de Salud en Colombia? Bogotá, Colombia.

Alvesson, M. \& Willmott, H. (2002). Identity regulation as organizational control. Producing the ap- propiate individual. Journal of Management Studies, 39 (5), 619-644.

Arroyo, J. (2002). Situación y desafíos en el campo de los recursos humanos en salud en el área andina, en los albores de los 2000. Anales de la Facultad de Medicina. Universidad Nacional Mayor de San Marcos, 63 (3), 212-222.

Beck, U. (2000). Un nuevo mundo feliz. La precariedad del trabajo en la era de la globalización. Buenos Aires: Paidós.

Calderón-Hernández, G., Naranjo-Valencia, J. C. \& Álvarez-Giraldo, C. M. (2006). La gestión humana en Colombia: características y tendencias de la práctica y de la investigación. Estudios Gerenciales, 23 (103), 39-64.

Castel, R. (1997). La Metamorfosis de la cuestión social: una crónica del salariado. Buenos Aires: Paidós.

Castel, R. (2004). La inseguridad social iqué es estar protegido? Buenos Aires: Ediciones Manantial.

Cho, J. \& Trent, A. (2006). Validity in qualitative research revisited. Qualitative Research, 6 (3), 319-340.

Cunliffe, A. L. (2003). Reflexive inquiry in organizational research: Questions and possibilities. Human Relations, 56 (8), 983-1003.

Dávila, C. (1985). Teorías Organizacionales y Administración. Enfoque Crítico. Bogotá: Editorial Interamericana.

De Roux, F. (2010, noviembre). Reflexiones sobre la ética de la política publica. Ponencia presentada en el Primer Congreso Internacional de Sistemas de Salud ¿Hacia un Nuevo Sistema de Salud en Colombia? Bogotá, Colombia.

Flesher, J. (2009). The meaning of working. A perspective from practice. Advances in Developing Human Resources, 11 (2), 253-260.

Garavan, T. N., O’Donnell, D., McGuire, D. \& Watson, S. (2007). Exploring perspectives in human resource development: An introduction. Advances in Developing Human Resources, 8 (1), 3-10.

García-Álvarez, C. M. (2006). El hospital como empresa, nuevas prácticas, nuevos trabajadores. Universitas Psychologica, 6 (1), 146-154.

García-Álvarez, C. M. (2008). Subjetividades laborales: impacto de la reforma de la salud en trabajadores 
de tres hospitales públicos de Bogotá. Pre-til, 1 (6), 28-43.

García-Álvarez, C. M. \& Carvajal-Marín, L. M. (2007). Tecnologías empresariales del yo: la construcción de sujetos laborales en el contexto del trabajo inmaterial. Universitas Psychologica, 6 (1), 49-58.

Gergen, K. J. \& Thatchenkery, T. J. (1996). Organizational science as social construction: Postmodern potentials. The Journal of Applied Behavioral Science, 32 (4), 356-377.

Gillespie, R. (1993). Manufacturing knowledge: A history of the Hawthorne Studies. New York: Cambridge University Press.

Holton, E. F. (2003). Beyond incrementalism. What's the next paradigm for HRD? Human Resource Development Review, 2 (1), 3-5.

Legge, K. (2005). Human resource management. Rhetorics and realities. Nueva York: Palgrave.

Matallana, R. F., Amaya, J. L. \& Vásquez, M. E. (2008). Recursos humanos de la salud en Colombia. Balance, competencias y prospectiva. Bogotá: Javergraf.

Mayo, E. (1977). Problemas sociales de una civilización industrial. Buenos Aires: Nueva Visión.

McWilliams, A. D., Flete, V. \& Wright, P. (2001). Strategic management of human resources for global competitive advantage. Journal of Business Strategies, 18 (1), 1-24.

Miller-Franco, L., Bennett, S. \& Kanfer, R. (2002). Health sector reform and public sector health worker motivation: A conceptual framework. Social Science and Medicine, 54, 1255-1266.

Morgan, G. (1990). Imágenes de la organización. México: Alfaomega.

Naredo, J. M. (2001). Configuración y crisis del mito del trabajo. Archipiélago, 48, 13-24.

Papadopoulos, D. (2004). Psychology and the political [Editorial]. International Joumal of Critical Psychology, 12, 5-13.

Pulido-Martinez, H. C. (2008). On Psychology, work and the constitution of the subject: The case of the urban passenger transport system in Bogotá, Colombia. Tesis de doctorado sin publicar, Cardiff University, Cardiff, Gales.

Pulido-Martínez, H. C. (2009). Examinando las operaciones de la Psicología en su relación con el traba- jo. En M. A. Aguilar-Bustamente \& E. RenteríaPérez (Eds.), Psicología del Trabajo y de las Organizaciones. Reflexiones y Experiencias de Investigación (pp. 77-98). Bogotá: Universidad Santo Tomás.

Pulido-Martínez, H. C. (2010a). Autonomía en el trabajo. El reto por opciones a la luz de la producción del sujeto neo-liberal. Revista Iberoamericana de Psicología: Ciencia y Tecnología, 3 (1), 7-15.

Pulido-Martínez, H. C. (2010b). Psychological knowledge for the governance of the south. Critical Perspectives on Work, 6 (2/3), 177-189.

Pulido-Martínez, H. C. \& Carvajal-Marín, L. M. (2005). De la tradición a la racionalización. Repertorios culturales que circulan en una organización alimentadora del sistema de transporte masivo de pasajeros en Bogotá. Universitas Psychologica, 4 (3), 349-357.

Ramanujam, M. \& Rosseau, D. M. (2006). The challenges are organizational not just clinical. Journal of Organizational Behavior, 27 (7), 811-827.

Reilly, R. C. (2010). Found poems as a dimension of representational trustworthiness. Ponencia presentada en Qualitative Research in Management and Organization Conference. Alburqurque, Nuevo México, USA.

Restrepo-Jiménez, M. (1998). Comunicación para la dinámica organizacional. Prisma, 10, 80-87.

Ruiz, F., Matallana, M. A., Amaya, J. L., Vásquez, M. E., Parada, L. A. \& Piña, M. R. (2008). Recursos Humanos de la Salud en Colombia. Balance, competencias y prospectiva (3ed.). Bogotá: Javergraf.

Rose, N. (1992). Governing the enterprising self. In P. Heelas \& P. Morris (Eds.), The Values of the Enterprise Culture the Moral Debate (pp. 141-163). Londres: Routledge.

Rose, N. (1999). Governing the soul. The shaping of the private self. Londres: Free Association Books.

Schvarstein, L. (2003). La inteligencia social de las organizaciones: desarrollando las competencias necesarias para el ejercicio efectivo de la responsabilidad social. Buenos Aires: Paidós.

Stecher, A. (2010). El análisis critico del discurso como herramienta de investigación psicosocial del mundo del trabajo. Discusiones desde América Latina. Universitas Psychologica, 9 (1), 93-107. 
Urdaneta-Ballen, O. (2006). Psicología Organizacional aplicada a la gestión del capital humano. Bogotá: Panamericana.

Vega-Romero, R. (2008). Salud. La lección más importante de una experiencia de gobierno. Pre-til, 17 (6), 8-15.
Viteles, M. S. (1932). Industrial Psychology. New York: W.W. Norton \& Company, Inc.

Walkerdine, V. (2005). Freedom Psychology and neoliberal subject. Soundings, 25, 47-61. 
